\title{
ANÁLISE DO TRANSPORTE MUCOCILIAR DE TABAGISTAS EM INTERVALOS DE ABSTINÊNCIA DO TABACO
}

Guilherme Yassuyuki Tacao ${ }^{1}$, Ercy Mara Cípulo Ramos ${ }^{3}$, Paula Roberta da Silva Pestana ${ }^{2}$, Luiz Carlos Soares de Carvalho Junior ${ }^{2}$, Ana Paula Coelho Figueira Freire ${ }^{2}$, Dionei Ramos ${ }^{3}$

Universidade Estadual Paulista - FCT/UNESP. ${ }^{1}$ Fisioterapeuta, Presidente Prudente - SP. ${ }^{2}$ Mestrando, Presidente Prudente - SP. ${ }^{3}$ Professor, Presidente Prudente - SP. E-mail: guilhermetacao@yahoo.com.br - Apoio: FAPESP, PIBIC e INAIRA/CNPq

\section{RESUMO}

O tabagismo é considerado pela Organização Mundial da Saúde (OMS) a principal causa de morte evitável em todo o mundo. Portanto, o objetivo foi avaliar o efeito agudo do hábito tabagístico no transporte mucociliar em diferentes momentos. Foi composto por 2 grupos (Grupo tabagista n=20; e Grupo não tabagista $n=20$ ). Inicialmente foram obtidos dados pessoais, história tabágica e, após 12 horas em abstinência tabagística foi realizado teste de função pulmonar (espirometria), quantificação do monóxido de carbono no ar exalado (Coex) e mensuração do tempo de trânsito de sacarina (TTS). Para normalidade dos dados utilizou-se o teste de Shapiro Wilk, e para comparação entre grupos $t$ student ou Mann-Whitney, dependendo da distribuição. $O$ TTS mostrou valor inferior ao grupo controle, tendo a maior média encontrada após 20 minutos. 0 transporte mucociliar dos participantes tabagistas não foi diferente do grupo controle, mesmo após exposição aguda aos componentes do cigarro.

Palavras-chave: Transporte mucociliar, tabagismo, sintomas de abstinência

\section{ANALYSIS OF MUCOCILIARY CLEARANCE SMOKERS IN INTERVALS OF SMOKING ABSTINENCE}

\begin{abstract}
The smoking is considered by the World Health Organization (WHO), the main cause of preventable death in the world. Therefore, the objective was to evaluate the acute effect of smoking habit on mucociliary clearance at different times. It was composed of two groups (Smokers Group $\mathrm{n}=20$ and Nonsmoker Group $\mathrm{n}=20$ ). At first, personal data and smoking history were obtained, and after 12 hours in implementing smoking abstinence was performed pulmonary function test (spirometry), measuring carbon monoxide in exhaled air (Coex) and measurement of the transit time of saccharin (TTS). For normality of the data we used the Shapiro Wilk test, and for comparison between groups $t$ Student or Mann-Whitney test, depending on the distribution. The TTS was lower to the control group, having average found after 20 minutes. The mucociliary clearance of the smokers participants was not different from control group, even after acute exposure to cigarette components.
\end{abstract}

Keywords: Mucociliary clearance, smoking, abstinence symptoms. 


\section{INTRODUÇÃO}

O tabagismo é considerado pela Organização Mundial da Saúde (OMS) a principal causa de morte evitável em todo o mundo. A OMS estima que um terço da população mundial adulta, isto é, 1 bilhão e 200 milhões de pessoas sejam fumantes ${ }^{(1)}$. Se não forem instituídas medidas eficazes de prevenção e controle, o número de mortes tende a aumentar para mais de oito milhões ao ano em 2030 , e mais de $80 \%$ dessas mortes devem ocorrer nos países em desenvolvimento. ${ }^{(2)}$

As substâncias tóxicas e irritantes presentes na fumaça do cigarro levam a um remodelamento epitelial com aumento do número de células caliciformes e hipertrofia das células mucosas, as quais propiciam um aumento considerável na produção de muco e geram prejuízo ao transporte mucociliar ${ }^{(3,4)}$ com consequente acúmulo de secreção e instalação de processos inflamatórios da mucosa brônquica, levando a um aumento de morbidade por doenças respiratórias ${ }^{(5)}$.

O transporte mucociliar é o principal mecanismo de defesa contra os agentes patogênicos e toxinas das vias aéreas superiores e inferiores. Entretanto, a eficiência do mesmo pode variar em diferentes condições, como à exposição a partículas nocivas presentes no fumo do cigarro $^{(6,7)}$.
Além dos benefícios da cessação do tabagismo para a saúde, as consequências relatadas sobre o efeito do cigarro no organismo ainda precisam ser melhores investigados. Sendo assim, buscamos encontrar novos e eficazes resultados para melhorar a qualidade de vidas dos indivíduos tabagistas.

Nesse contexto, os efeitos imediatos do transporte mucociliar após a cessação tabágica são importantes, pois é um mecanismo de defesa que elimina as toxinas das vias aéreas superiores e inferiores. Desta forma, a importância do presente estudo é avaliar os efeitos imediatos da abstinência tabagística em curtos períodos de tempo através do Tempo de Trânsito de Sacarina (TTS).

\section{METODOLOGIA}

Este estudo longitudinal avaliou 40 indivíduos, que foram divididos em dois grupos. O grupo tabagista foi composto por 20 indivíduos tabagistas (10 homens; $46 \pm 13$ anos; $17 \pm 8$ cigarros/dia; $26 \pm 22$ anos/maço), e o grupo controle por 20 indivíduos nãotabagistas (9 homens; $46 \pm 12$ anos)

Os indivíduos foram previamente orientados sobre os objetivos e procedimentos da pesquisa e foram incluídos na pesquisa após fornecerem o consentimento por escrito, com a assinatura de um termo de consentimento livre e 
esclarecido. O estudo foi aprovado pelo Comitê de Ética em Pesquisa da Instituição (parecer no: 032/2009).

Foram excluídos do estudo indivíduos com diagnóstico clinico prévio de doenças pulmonares, doenças tabaco relacionadas, comprovadas pela prova de função pulmonar, histórico de infecções respiratórias e internações hospitalares recentes.

No protocolo experimental do estudo, todos os indivíduos foram entrevistados para obtenção de dados pessoais, antropométrico e histórico tabagístico (tempo de tabagismo, número de cigarros/dia, índice anos/maço e grau de dependência de nicotina - Teste de Fagerström ${ }^{(8)}$. Em seguida, foram avaliados quanto à função pulmonar (espirometria), quantificação do monóxido de carbono no ar exalado (COex) e mensuração do transporte mucociliar pelo teste de Tempo de Transito de Sacarina (TTS).

As avaliações foram realizadas no período da manhã no ambulatório de Fisioterapia em quatro momentos. No primeiro momento foram realizados a entrevista, espirometria, quantificação do COex e mensuração do TTS em 12 horas de abstinência para quantificação do TTS basal dos indivíduos tabagistas. No segundo, terceiro e quarto momentos, foram realizadas quantificações do COex e a mensuração do TTS imediatamente, 20 minutos e 120 minutos após o ato de fumar. Os voluntários foram orientados a não fazer uso de medicamentos tais como anestésicos, analgésicos barbitúricos, calmantes e antidepressivos; de bebidas alcoólicas e de substâncias a base de cafeína no mínimo 12 horas antes da mensuração do TTS.

Para a mensuração do transporte mucociliar nasal foi utilizado o teste de tempo de trânsito de sacarina (TTS), onde foi introduzida por meio de uma cânula plástica, sob controle visual, $5 \mu \mathrm{g}$ de sacarina sódica granulada a aproximadamente $2 \mathrm{~cm}$ dentro da narina direita. ${ }^{(9)}$

A prova de função pulmonar foi realizada pelo espirômetro da marca MIRSpirobank versão 3.6, previamente calibrado. Foi realizada a manobra de Capacidade Vital Forçada (CVF) e analisado os valores absolutos da relação do Volume Expiratório Forçado no 1ㅇ segundo sobre Capacidade Vital Forçada (VEF1/CVF) e os percentuais dos valores preditos do VEF1. Os valores previstos foram obtidos a partir dos valores de referência da população brasileira ${ }^{(10)}$.

Para o teste de monóxido de carbono no ar exalado (COex) por técnica padronizada (aparelho MicroCO Meter, Micro Medical Ltd., Rochester, Kent, Reino Unido) para confirmação do período de abstinência solicitado. $^{(11,12)}$ 


\section{ANÁLISE ESTATÍSTICA}

Para a análise estatística, os dados foram analisados pelo programa SigmaStat 3.0 (Inc., San Diego CA, USA). A normalidade na distribuição dos dados foi avaliada por meio do teste de Shapiro-Wilk. A descrição dos resultados foi realizada como média e desvio padrão. Para comparação entre os grupos foi utilizado teste $t$ student ou teste Mann-Whitney dependendo da normalidade da distribuição dos dados. A significância estatística foi determinada como $p<0,05$.

\section{RESULTADOS}

Tabela 1. Características antropométricas dos grupos de tabagistas e não tabagistas. Dados apresentados em média e desvio padrão.

\begin{tabular}{llc}
\hline & Tabagistas $(\mathrm{n}=20)$ & Não Tabagistas $(\mathrm{n}=20)$ \\
Idade (anos) & $46 \pm 13$ & $46 \pm 12$ \\
IMC $\left(\mathrm{kg} / \mathrm{m}^{2}\right)$ & $26 \pm 4$ & $26 \pm 4$ \\
Sexo $(\mathrm{M} / \mathrm{F})$ & $10 / 10$ & $9 / 11$ \\
Consumo de cig/dia & $17 \pm 08$ & - \\
Tempo de fumo (anos) & $24 \pm 14$ & - \\
Índice anos/maço & $26 \pm 22$ & - \\
Dependência de nicotina & $5 \pm 8$ & - \\
VEF1\% & $93 \pm 27$ & $100 \pm 11$ \\
\hline
\end{tabular}

Legenda: $\mathrm{F} / \mathrm{M}=$ feminino/masculino; $\mathrm{kg}=$ kilogramas; $\mathrm{m}=$ metros; $\mathrm{IMC}=$ índice de massa corporal; $\mathrm{VEF}_{1}=\mathrm{Volume}$ expiratório forçado no primeiro segundo; TTS= Tempo de Trânsito de Sacarina min= minutos.

As medidas dos valores de monóxido de carbono no ar exalado (COex) estão apresentadas na figura 1 . Os valores de COex dos indivíduos do grupo tabagista eram significantemente maiores em relação ao grupo controle em todos os períodos analisados. A maior média de COex foi obtida pelo grupo tabagista foi no momento dos indivíduos avaliados estão apresentadas na tabela 1 . Todos os indivíduos incluídos no estudo apresentaram função pulmonar normal, segundo os critérios estabelecidos pelas Diretrizes para Testes de Função Pulmonar ${ }^{(13,14)}$. Os voluntários dos grupos tabagistas e não tabagistas foram semelhantes quanto aos dados de idade e IMC $(p \leq 0,05)$. Os indivíduos tabagistas foram classificados como tabagistas moderados de acordo com o consumo de cigarros/dia ${ }^{(15)} \mathrm{e}$ com dependência moderados a nicotina, medida por meio do teste de Fagerström. imediatamente após fumar, $18 \pm 9 \mathrm{ppm}$, e a menor no momento após 12 horas de abstinência, 11 \pm 7 ppm. (Figura 1)

Os valores de tempo de trânsito de sacarina (TTS) estão descritos em minutos (min) por meio de média e desvio padrão. Não houve diferenças estatisticamente significantes nos valores de TTS entre os 
grupos controle e tabagista em 12 horas de abstinência. Também não houve diferença estatisticamente significante na comparação dos momentos de avaliação, imediato, 20 minutos e 120 minutos, quando comparados a 12 horas de abstinência. A maior média de TTS foi encontrada após 20 minutos do ato de fumar $(9 \pm 4 \mathrm{~min})$, e menor valor imediatamente após $\mathrm{o}$ ato de fumar (7 \pm 3 min). $(p=0,39)$. (Figura 2$)$

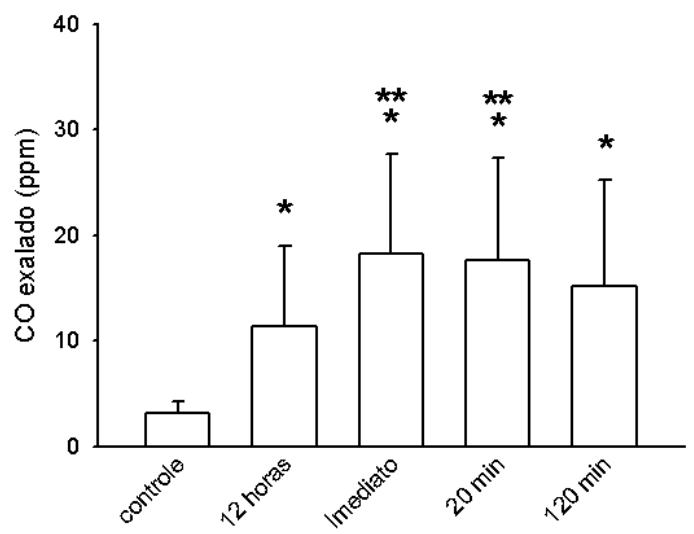

Figura 1. Monóxido de Carbono no ar exalado (COex) dos indivíduos tabagistas nos quatro períodos analisados e não tabagistas. Dados apresentados em partes por milhão (ppm) em média \pm desvio padrão. ${ }^{*} p<0,001$ em relação ao controle; ${ }^{* * *} p<0,01$ em relação ao grupo 12 horas.

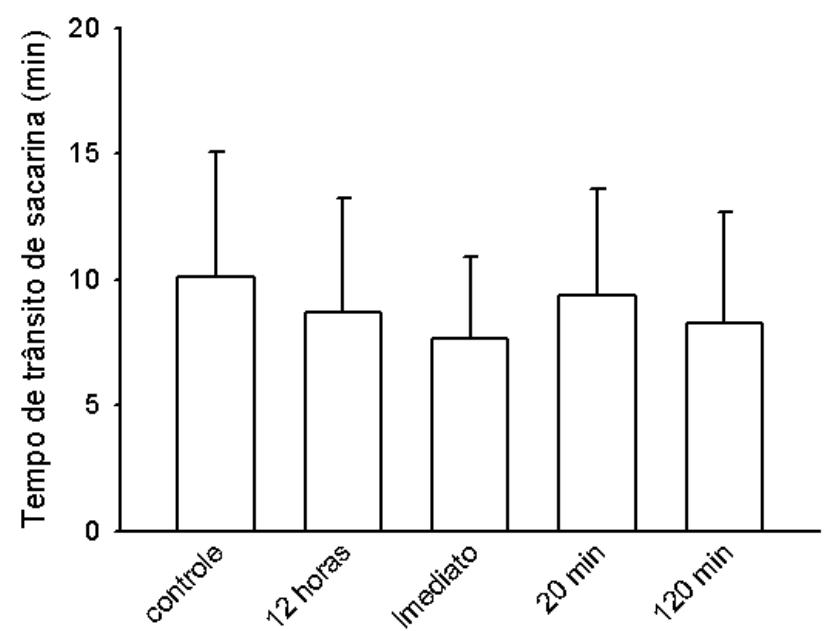

Figura 2. Tempo de Trânsito de Sacarina nos quatro períodos analisados, 12 horas de abstinência, imediatamente, 20 minutos e 120 minutos após fumar, de indivíduos tabagistas do grupo controle em média \pm desvio padrão.

\section{DISCUSSÃO}

O presente estudo mostrou que, não houve diferenças significativas em diferentes períodos de abstinência em relação à quantificação do TTS em indivíduos tabagistas e não tabagistas. Entretanto, a 
maior média do tempo foi encontrada após 20 minutos do ato de fumar e menor valor imediatamente após o ato de fumar. Tais resultados podem ser atribuídos ao fato de que, a fumaça do cigarro produz uma redução no batimento ciliar após estímulo. (16)

Da mesma forma, não houve resposta aguda no transporte mucociliar de indivíduos tabagistas imediatamente após a exposição à fumaça de cigarros e o mesmo ocorreu entre os tempos 20 e 120 minutos após a exposição. Portanto, um TTS próximo aos valores de normalidade ${ }^{(17)}$ encontrados em tabagistas imediatamente após o fumo pode estar relacionado com o sistema nervoso simpático, causando uma possível aceleração do batimento ciliar ${ }^{(18)}$.

A diferença entre 0 TTS imediatamente e após o ato de fumar pode ser um efeito da nicotina sobre o sistema nervoso autônomo (SNA), tal condição é representada devido à inervação motora, sensorial e autônoma na região nasal, devendo ressaltar que, essa substância provoca estimulação simpática neural ocasionando uma ativação do metabolismo geral do corpo. Em contrapartida, o sistema nervoso parassimpático está relacionado com os receptores nicotínicos de acetilcolina, que se encontram aumentados em tabagistas crônicos $^{(19)}$.
Entretanto, a nicotina circulante é metabolizada em duas horas, sendo assim, o efeito estimulante cessa e o batimento ciliar retorna ao seu basal. Essa reversibilidade prejudicada em fumantes pode ser exemplificada no estudo de Ramos et al. ${ }^{(20)}$, onde a cessação tabagística após 15 dias contribuiu para uma melhora do transporte mucociliar e os benefícios persistiram por mais 180 dias. Rodrigues et al. ${ }^{(21)}$, também afirmaram que, a abstinência tabagística a curto prazo diminui a inflamação sistêmica e melhora o transporte mucociliar, porém não tendo diferenças na inflamação nasal.

Stanley et al. ${ }^{(22)}$, compararam o TTS em fumadores e não fumadores, e também concluíram que o tempo dos fumadores estão aumentados em relação aos não fumadores, não tendo diferenças nas médias da frequência do batimento ciliar. Em contrapartida, Baby et al. ${ }^{(23)}$, relata uma diminuição do batimento ciliar, redução do número de cílios e mudança na propriedade viscoelástica do muco em fumantes e não fumantes. Este mecanismo de defesa é afetado por substâncias presentes na fumaça do cigarro e seu funcionamento adequado depende da interação entre os cílios e o muco.

Apesar de poucos trabalhos encontrados na literatura em relação à abstinência e o transporte mucociliar, as diferenças entre os valores de TTS de 
indivíduos normais encontrados devem-se provavelmente aos diversos protocolos e tipos de populações aplicadas. Por fim, destacamos os achados deste estudo acrescentam novas informações à escassa literatura referente à abstinência e sua relação com o transporte mucociliar em fumadores, especialmente a resposta aguda deste mecanismo.

\section{CONCLUSÃO}

O transporte mucociliar nasal de indivíduos tabagistas após 12 horas de abstinência não foi diferente de indivíduos saudáveis e não houve alteração no transporte mucociliar nasal de indivíduos tabagistas após exposição aguda as substâncias do cigarro.

\section{AGRADECIMENTOS}

Esse trabalho foi financiado pela Fundação de Amparo à Pesquisa do Estado de São Paulo (FAPESP), o Programa Institucional de Bolsas de Iniciação Científica - PIBIC. Os autores declaram não haver qualquer potencial conflito de interesse que possa interferir na imparcialidade deste trabalho científico.

\section{REFERÊNCIAS}

1. Saúde. Ministério da Saúde.[Site da Internet] Tabagismo: Dados e números. Disponível http://www.inca.gov.br/tabagismo/frameset. asp?item =dadosnum\&link=mundo.htm

2. Who - Report on the Global Tobacco Epidemic, 2008: The MPOWER package. Geneva: World Health Organization; 2008.

3. Nunes E. Consumo de tabaco. Efeitos na saúde. Revista Portuguesa de Clínica Geral 2006; 22(44): 225-44.

4. Koczulla AR. et al. Acute and Chronic Effects of Smoking on Inflammation Markers in Exhaled Breath Condensate in Current Smokers. Basic Science Investigations Respiration. 2010; 79:61-67. http://dx.doi.org/10.1159/000245325

5. Elliot MK, Sisson JH, Wyatt TA. Effects of Cigarette Smoke and Alcohol on Ciliated Tracheal Epithelium and Inflammatory Cell Recruitment. American Journal of Respiratory Cell and Molecular Biology 2007; 36:452-459. http://dx.doi.org/10.1165/rcmb.200504400C

6. Nakagawa NK, Franchini ML, Driusso P, de Oliveira LR, Saldiva PH. G. Mucociliary clearance is impaired in acutely ill patients. Chest. 2005; 128:2772-7. http://dx.doi.org/10.1378/chest.128.4.2772

7. Stannard W, O'Callaghan C. Ciliary function and the role of cilia in clearance. Journal Aerosol Medicine. 2006; 19:110-5. http://dx.doi.org/10.1089/jam.2006.19.110

8. Do Carmo JT \& Pueyo AA. A adaptação ao Português do Fagerström Test for Nicotine dependence (FTND) para avaliar a dependência e a tolerância à nicotina em fumantes brasileiros. Revista Brasileira de Medicina. 2002; 59: 73-80.

9. Andersen, JB, Camner P., Jensen PL, Philipson K, Proctor DF. A comparison of nasal and tracheobronquial clearance. 
Arquives of Enviromental Health 1974; 29: 290-93.

http://dx.doi.org/10.1080/00039896.1974.10 666589

10. Pereira CAC, Taeko S, Rodrigues SC. Novos valores de referência para espirometria forçada em brasileiros adultos de raça branca. Jornal Brasileiro de Pneumologia 2007. 33(4): 397-406. http://dx.doi.org/10.1590/S180637132007000400008

11. Chatkin, G; Chatkin, JM; Aued, G; Petersen, GO; Jeremias, ET; Thiesen, FV. Avaliação da concentração de monóxido de carbono no ar exalado em tabagistas com DPOC. Jornal Brasileiro de Pneumologia;36(3):332-338, maio-jun. 2010. http://dx.doi.org/10.1590/S180637132010000300011

12. Chatkin JM, Abreu CM, Haggstram FM, Blanco DC, Rodini V, Martins D, et al. Exhaled CO: is $10 \mathrm{ppm}$ a reliable threshold value to confirm current smoking? American Journal Respiratory Critical Care Medicine. 2002;165:19s.

13. Sociedade Brasileira de Pneumologia e Tisiologia. Diretrizes para testes de função pulmonar. Jornal Brasileiro de Pneumologia. 2002;28(supl 3):S44-S58.

14. Rubin AS, Cavalazzi AC, Viegas CAA, Pereira CAC, Nakaie CMA, Valle ELT. Et al Diretrizes para testes de função pulmonar. Jornal Brasileiro de Pneumologia 2002; 28(3): 1-82

15. Trosclair A, Dube SR. Smoking among adults reporting lifetime depression, anxiety, anxiety with depression, and major depressive episode, United States, 20052006. Additive Behaviors 2010; 35: 4380443. http://dx.doi.org/10.1016/j.addbeh.2009.12. 011
16. Kimura BC, et al. Efeito do Tabagismo no Transporte Mucociliar Nasal. Disponível em:

$<$ https://uspdigital.usp.br/siicusp/cdOnlineTr abalhoVisualizarResumo?numerolnscricaoTra balho $=4058 \&$ numeroEdicao $=16>$

17. Valía PP, Valero FC, Pardo JM, Rentero DB, Monte CG. Saccharin Test for the Study of Mucociliary Clearance: Reference Values for a Spanish Population. Archives of Bronconeumology 2008; 44(10): 540-5. http://dx.doi.org/10.1016/S1579-

2129(08)60100-7

18. Rappai M, Collop N, Kemp S, deShazo R. What we know and what we do not know the nose and sleep-disordered breathing: what we know and what we do not know. Chest. 2003;124:2309-23. http://dx.doi.org/10.1378/chest.124.6.2309

19. Yuna AJ, Bazarb AK, Leec PY, Gerber A, Daniel SM. The smok- ing gun: many conditions associated with tobacco exposure may be attributable to paradoxical compensatory auto- nomic responses to nicotine. Medical Hypotheses. 2005;64: 1073-9.

http://dx.doi.org/10.1016/j.mehy.2004.11.04 0

20. Ramos EMC, et al. Reversibility of impaired nasal mucociliary clearance in smokers following a smoking cessation programme. Journal of the Asian Pacific Society of Respirology 2011; 16, 849-855. doi: 10.1111/j.1440-1843.2011.01985.x

21. Rodrigues, FMM et al. Nasal and systemic inflammatory profile after short term smoking cessation. Respiratory Medicine (2014), 108, 999-1006. http://dx.doi.org/10.1016/j.rmed.2014.04.02 0

22. Stanley PJ, Wilson R, Greenstone MA, MacWilliam L, Cole PJ. Effect of cigarette 
smoking on nasal mucociliary clearance and ciliary beat frequency. Thorax. 1986;41:51923. http://dx.doi.org/10.1136/thx.41.7.519

23. Baby MK et al. Effect of cigarette smoking on nasal mucociliary clearance: A comparative analysis using saccharin test. Lung India (2014), vol 31, Issue 1.

Recebido para publicação em 18/08/2014

Revisado em 12/09/2014

Aceito em 15/09/2014 